\title{
Resonant diffraction study of structural disorder in $\mathrm{Nb}_{3} \mathrm{Sn}$
}

$\underline{\text { Roman Svetogorov }}^{1}$, Yan Zubavichus ${ }^{1}$, Sergey Shavkin ${ }^{1}$, Alexander Ryazanov ${ }^{1}$, Rene Flukiger ${ }^{2}$, Evgeny Semenov ${ }^{1}$, Luca Bottura ${ }^{2}$, Christian Scheuerlein ${ }^{2}$

${ }^{1}$ NRC Kurchatov Institute, Moscow, Russian Federation, ${ }^{2}$ CERN, Geneve, Switzerland

E-mail: rdsvetov@gmail.com

$\mathrm{Nb} 3 \mathrm{Sn}$ based low-temperature superconductors have excellent critical characteristics to be widely used, for example, in superconducting coils that generate ultrastrong magnetic fields in different particle accelerators. During the lifetime such coils are constantly exposed to high energy particles that may alter their structure and respectively give rise to a degradation of the superconducting properties. One of the major types of structural defects arising in Nb3Sn under the influence of particle irradiation and strongly affecting electrophysical characteristics is antisiting, which implies that niobium and tin partially swap their positions in the crystal lattice. The extent of this disorder is typically quantified by the BraggWilliams long-range atomic order (LRO) parameter S [1]. The variations of the critical parameters of A15 compounds (TC, Bc) in terms of the degree of atomic order have been studied earlier [2], and it was established that for all typical A15-type superconductors a decrease in Tc and Bc strongly correlates with the decrease in S. Therefore the problem of accurate evaluation of the LRO parameter becomes very important.

A simple way to obtain $S$ consists in using the Rietveld refinement of X-ray powder patterns. The LRO parameter is calculated from the atomic occupation factors that are contained in the structure amplitudes. Unfortunately there are some problems in the use of that straightforward approach, since the disorder is usually minute and Nb and $\mathrm{Sn}$ atoms are not that far in the Periodic Table. Furthermore, even weak preferred orientation changes relative intensities applreciably. Thus the Rietveld refinement often yields unphysical values (e.g. zero LRO parameter). A more potent method of determining the Bragg-Williams parameter consists may rely on the effect of resonant (or anomal) scattering. The resonant X-ray diffraction (REXD) method is based on the measurement of a number of powder diffraction patterns in the proximity of an absorption edge of one of the elements in the compound. For the precise determination of $S$ it is practical to acquire a series of energydependent diffraction patterns over a reasonably wide photon energy range near the K-edge of niobium. Then by solving corresponding equations for the experimentally obtained intensity, one gets the desired values of the Bragg-Williams parameter with a high accuracy.

Nb3Sn samples studied within the project were synthesized and provided to NRC "Kurchatov Instittue" by René Flükiger and co-workers, CERN, Switzerland. This set included a couple of plate-like polycrystalline samples with thicknesses of 150 - 300 microns that were measured and then irradiated by fast protons with an energy of 12 and 35 MeV on the cyclotron U-150 NRC "Kurchatov Institute" at the room temperature. Powder diffraction experiments were carried out at the "Structural materials science" beamline of the Kurchatov synchrotron radiation source (NRC "Kurchatov Institute", Moscow, Russia) [3].

[1] W. Bragg and E. J. Williams (1934) Proc. R. Soc. Lond. A. 145, 699-730.

[2]R. D. Blaugher et. al. (1969) J Low Temp Phys. 1, 6, 539-561.

[3] A.A. Chernyshov et al. (2009) Nucl Instrum Methods Phys Res A. 603, 95-98

Keywords: Synchrotron, Resonant diffraction, Superconductors 